\title{
Low Molecular Weight Z-Tetraol Boundary Lubricant Films in Hard Disk Drives
}

\author{
R. J. Waltman and H. Deng \\ Media Development, HGST Western Digital, 5601 Great Oaks Parkway, San Jose, CA 95119-1003, USA \\ Correspondence should be addressed to R. J. Waltman, robert.waltman@hitachigst.com
}

Received 16 August 2012; Accepted 5 November 2012

Academic Editor: Bruno Marchon

Copyright ( $) 2012$ R. J. Waltman and H. Deng. This is an open access article distributed under the Creative Commons Attribution License, which permits unrestricted use, distribution, and reproduction in any medium, provided the original work is properly cited.

Lower molecular weight Z-Tetraol films exhibit increased mechanical spacing in the slider-disk interface due to a lower z-profile. An increased resistance to lubricant disturbance on the disk surface (e.g., lube moguls) with decreasing film thickness is attributed to an increasing contribution from the polar component of the disjoining pressure. Evaporative loss at temperatures typically encountered in a hard-disk drive also increases with decreasing molecular weight but is strongly dependent on the initial bonded fraction.

\section{Introduction}

In order to keep pace with the higher areal storage density requirements of hard-disk drives (HDDs), the spacing between the read-write element of the slider and the surface of the rigid magnetic disk has been reduced to 1-2 nm. Perfluoropolyether (PFPE) boundary lubricant films having reduced dynamic expansion perpendicular to the disk surface ("low z-profile") have allowed lower flying. Low zprofile PFPEs can be attained by reducing the film thickness, the main chain molecular weight, and its flexibility [1-3]. Based on these studies, a useful mnemonic relating sliderdisk mechanical clearance, $\Delta$ clearance, to these PFPE main chain properties is given in (1) as follows:

$$
\Delta \text { clearance } \propto \frac{1}{\left(\mathrm{MW}^{1 / 2}\right)(h)(f)} .
$$

MW is the molecular weight, $h$ is the film thickness, and $f$ represents the main chain flexibility.

In this report, we compare the boundary lubrication properties of lower MW Z-Tetraols for their potential use in HDDs. Thus the effect on mechanical clearance, surface energy, lubricant dewetting, and lubricant evaporation at drive temperatures is quantified.

\section{Experimental}

The substrates used in these studies were $65 \mathrm{~mm}$ diameter glass disks of nominally $2 \AA$ RMS roughness as measured by a Dimension 5000 AFM using a standard AFM tip in the tapping mode. The typical scan size for these measurements was $1 \mu \mathrm{m} \times 1 \mu \mathrm{m}$ with a scan rate of $0.5 \mathrm{~Hz}$ and 256 lines of resolution. Atop the substrates a cobalt-based magnetic recording layer ( $\mathrm{CoPtCr}$ ) was sputter deposited, followed by CVD deposition of $30 \AA$ of an amorphous nitrogenated carbon film (called " $\mathrm{CN}_{x}$ ") comprised of nominally 15 at $\%$ N. Z-Tetraol-GT of 1200 and 2200 dalton (MW) were purchased from Solvay-Solexis (Italy) and used directly (Figure 1). The NMR compositions of the samples are quantified in Table 1.

Specular reflection FTIR (Nicolet Magna Model 560, Thermo Fisher) was used to quantify the film thicknesses of the applied PFPEs. The main IR absorption band near $1280 \mathrm{~cm}^{-1}$, due to the combination of C-F and C-O stretching vibrations, was related to film thickness as determined by XPS (Physical Electronics Instruments) [4]. Thin films from 0.7 to $1.8 \mathrm{~nm}$ provided the film thickness calibration. A standard (XPS) takeoff angle $\left(45 \pm 15^{\circ}\right)$ and an electron mean-free path of $2.5 \mathrm{~nm}$ were used to determine the PFPE film thicknesses. 


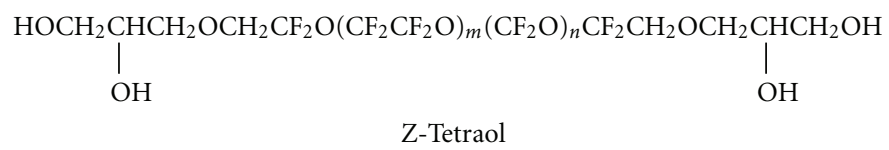

FIGURE 1: Chemical structure of Z-Tetraol.

TABLe 1: NMR data for the PFPE lubricants.

\begin{tabular}{lcccc}
\hline Lubricant & Mn & Tetraol & dol & bis \\
\hline Z-Tetraol 1200 & 1200 & 0.98 & 0.02 & 0.00 \\
Z-Tetraol 2200 & 2200 & 0.95 & 0.05 & 0.00 \\
\hline
\end{tabular}

The mechanical clearance of the PFPE lubricants was quantified by measuring the acoustic emission (AE) generated at the head-disk interface as a function of sliderdisk clearance. In these experiments, lubricated disks were placed on a spin stand and rotated at a constant 7200 RPM under ambient conditions. At the start of the experiments, the slider was allowed to fly over the rotating disk surface at a height of $\sim 8 \mathrm{~nm}$, and the slider was heated to protrude the read-write element, that is, pole tip protrusion (PTP). During this procedure, the $\mathrm{AE}$ signal was continuously monitored, and the onset of an AE signal was interpreted as the occurrence of slider-disk interaction. The slider fly height versus (thermal fly height control) TFC power was used to calibrate the clearance based on the touchdown detection. The measurements were interleaved to minimize test variables. Each experiment was repeated 12-14 times, and the reproducibility of the data, despite the fairly large distribution, was taken as evidence that the slider flying characteristics were constant.

Lubricant moguls were induced on the disk surface by flying a slider on-track at a fly-height of $8 \mathrm{~nm}$ with no pole tip protrusion, under ambient conditions. A Vena VS-90 spinstand tester was used. After 20 min of on-track flying, the disks were immediately interrogated by an optical surface analyzer (Q-phase image using a Candela Model 6120) and the lubricant mogul thicknesses were quantified.

\section{Results}

Representative data showing the $\mathrm{AE}$ signal as a function of the slider-disk clearance is shown in Figure 2(a) for Z-Tetraol 2200 and 1200 MW. A summary plot showing the average and distribution of all clearance data is shown in Figure 2(b) where the clearance values are plotted relative to $11.5 \AA \mathrm{Z}$ Tetraol 2200. A positive clearance value therefore represents an increase or a gain in mechanical clearance. The lower MW Z-Tetraol shows approximately $0.8 \mathrm{~nm}$ improved clearance between the slider and disk surfaces, and this result is fully expected on the basis of (1); that is, a reduction in MW and a slightly thinner film (by $0.5 \AA$ ) allows lower flying before onset of slider-disk interference. The contribution to the clearance gain from the $0.5 \AA$ film thickness difference is deconvolved from the inset figure $(\sim 0.6 \mathrm{~nm} / \AA)$ to provide $\sim 0.5 \mathrm{~nm}$ clearance gain by the Z-Tetraol MW reduction from
2200 to 1200 . The details are provided in the caption of Figure 2.

Figure 3 shows the lubricant disturbance on the disk surface as a function of MW and film thickness after headflying. The lubricant disturbance leads to an accumulation of lubricant material in excess of the nominal film thickness that is shown as dark spots on the disk surface, and these are referred to as "lube moguls" [5]. The lighter spots in the images represent areas on the disk surface that has lost lubricant film thickness presumably to the moguls. It appears that the Z-Tetraol 1200 exhibits less lube moguls than the ZTetraol 2200 and may originate from the increased clearance (Figure 2). The reduction in lube moguls as a function of decreasing film thickness (Figure 3 ) has been attributed to increased adhesion with decreasing film thickness [6].

\section{Discussion}

According to (1), mechanical clearance from PFPE lubricants may be harvested by decreasing: (a) the film thickness, (b) the MW, and (c) the main chain flexibility. The extent to which each of these adjustments can be utilized is also limited because they can impact the HDD interface deleteriously. For example, reducing the film thickness to $\leq \sim 8 \AA$ increases the surface energy significantly (Figure 4 ) thereby increasing the probability for contamination adsorption in the HDD. Since the focal point of this paper is the effect of MW reduction as a means to gain mechanical clearance, limitations attributable to decreasing the MW are now discussed.

Valuable information on PFPE MW effects is readily realized by measuring the surface energy as a function of film thickness and of MW. Information relevant to the adhesive strength, propensity for contamination adsorption, the lubricant monolayer thickness, and thus the critical dewetting thickness may be quickly assessed. Figure 4(a) shows the dispersive surface energy, $\gamma_{s}^{d}$, as a function of film thickness for Z-Tetraols on $\mathrm{CN}_{x}$. The $\gamma_{s}^{d}$ decreases monotonically with increasing film thickness in all cases, approaching what is expected to be the bulk dispersive surface energy near $14 \mathrm{~mJ} / \mathrm{m}^{2}$. There does not appear to be a significant MW effect on $\gamma_{s}^{d}$. Quantitatively, the dependence of $\gamma_{s}^{d}$ on the applied lubricant film thickness $h$, that is, $\gamma_{s}^{d}(h)$, can be described using $\gamma_{s}^{d}(h)=\gamma_{1}^{d}+\Delta \gamma^{d}(h)$, where

$$
2 \Delta \gamma^{d}(h)=\frac{1}{12 \pi} \frac{A^{*}}{\left(d_{o}+h\right)^{2}} .
$$

$d_{o}$ is a constant, and $A^{*}$ is the "effective" Hamaker constant [7]. The $d_{o}$ values used to fit the dispersive surface energy curves via (1), $d_{0}=2.5 \pm 0.5 \AA$, is indicative that the interaction between the PFPE main chain and the underlying carbon film is relatively weak and hence cannot provide 


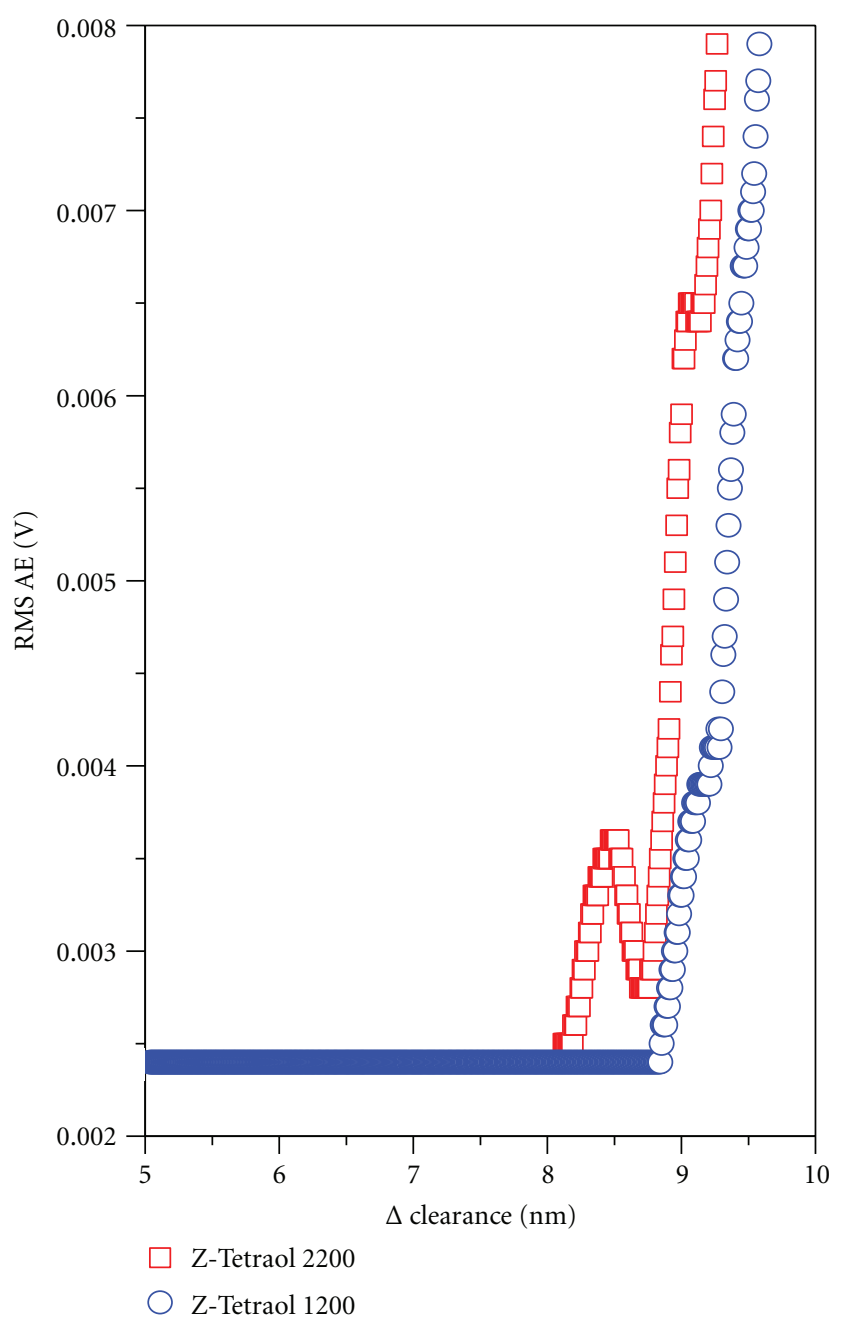

(a)

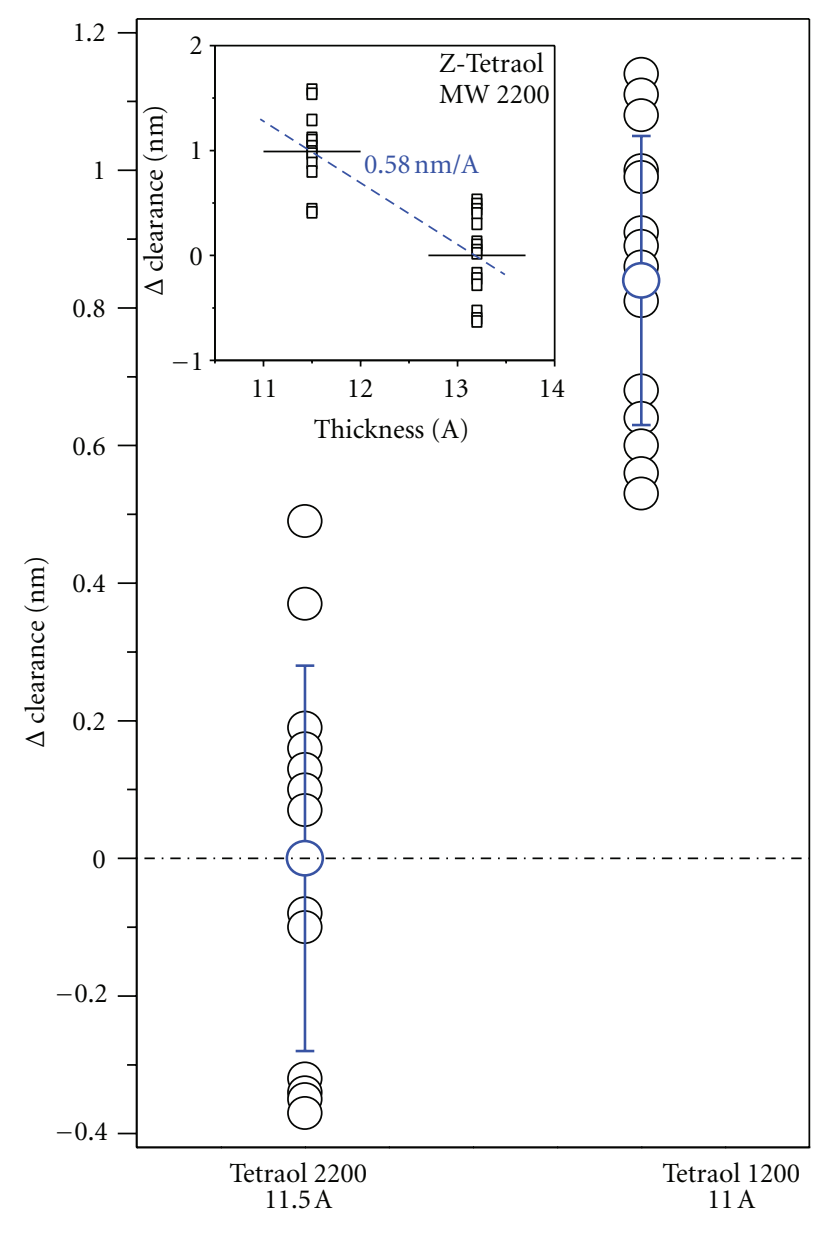

(b)

FIGURE 2: (a) Representative data plotting the rms acoustic emission (AE) as a function of slider-disk clearance for $11.5 \AA$ Z-Tetraol 2200 and $11.0 \AA \mathrm{Z}$-Tetraol 1200 . The $\Delta$ clearance is defined as the distance between the thermally protruding read-write element of the slider and the disk surface. (b) The slider-disk clearance data plotted relative to Z-Tetraol 2200. Open circles are the individual data points. Average data are represented by the blue points with 1 sigma standard deviation. The inset figure plots the $\Delta$ clearance as a function of film thickness for Z-Tetraol 2200, providing a slope of $0.58 \mathrm{~nm} / \AA$. Thus, approximately $0.3 \mathrm{~nm}$ of the clearance difference between $11.5 \AA \mathrm{Z}$-Tetraol 2200 and $11.0 \AA \mathrm{Z}$-Tetraol $1200 \mathrm{can}$ be attributed to the thickness difference.

significant adhesion. The corresponding $A^{*}$ are equal to $1.9 \times 10^{-19} \mathrm{~J}$ and $2.1 \times 10^{-19} \mathrm{~J}$ for Z-Tetral 2200 and $1200 \mathrm{MW}$, respectively. The larger $A^{*}$ for the lower MW ZTetraol is expected to provide a relatively better adhering film to the underlying carbon surface.

The corresponding polar surface energy, $\gamma_{s}^{p}$, as a function of film thickness is also shown in Figure 4(b). The distinct oscillations in $\gamma_{s}^{p}$ as a function of film thickness are indicative of molecular layering in the PFPEs induced by the polar interactions with the underlying surface, much as observed previously for Zdols [8]. As shown in the figure, the structuring is propagated through several or more monolayers. The first minimum corresponds to the film thickness at which the surface becomes saturated with lubricant, that is, the monolayer film thickness, which is approximately $13 \AA$ for $1200 \mathrm{MW}$ and $20 \AA$ for 2200 MW. Since HDDs today utilize
PFPE films near $10-12 \AA$, a reduction to a $1200 \mathrm{MW}$ may not provide enough of a thickness margin to prevent moguls formation under HDD conditions unless thinner films are utilized.

While the identification of the monolayer film thickness is important for optimizing the boundary lubricant film properties, its resistance to the effects of the low-flying slider including air shear effects and chemical and physical forces [9] is another important aspect that can be addressed by the surface energy measurements. Surface energy-derived disjoining pressures [10], computed in Figure 5 as a function of the Z-Tetraol MW, provide a good comparison of anticipated lubricant reliability in the HDD interface. In the submonolayer film thickness regime, the total disjoining pressure gradient is larger for the lower MW Z-Tetraol due to the comparatively larger contribution from its polar component. 


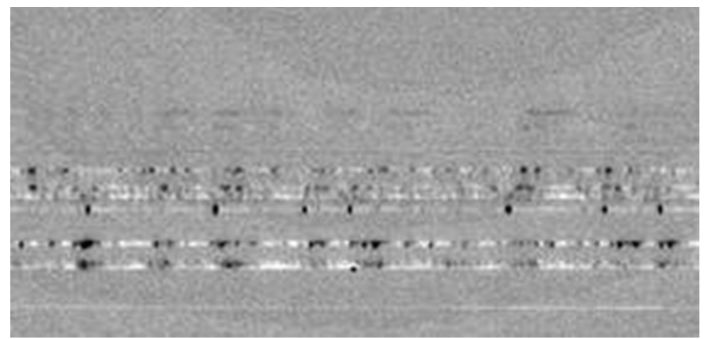

$4.5 \AA ̊$ moguls 2200

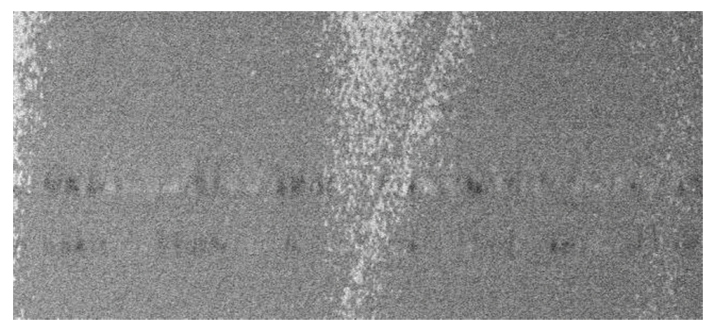

$1.5 \AA ̊$ moguls 1200

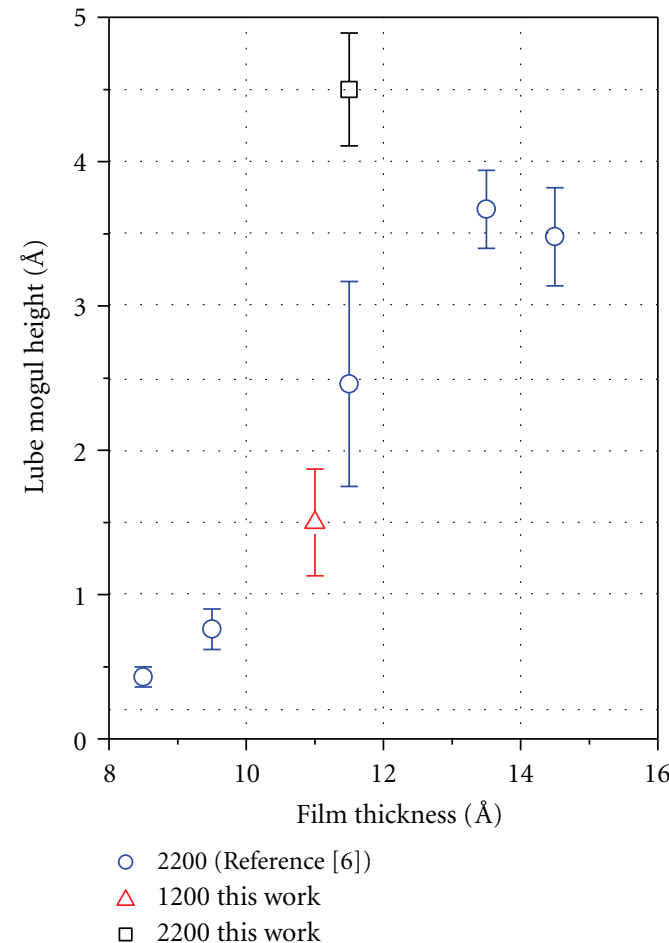

(b)

Figure 3: (a) Representative images of lube moguls after low flying on $11.5 \AA$ Z-Tetraol 2200 (top) and $11.0 \AA$ Z-Tetraol 1200 (bottom). These data were generated by flying the slider at $8 \mathrm{~nm}$ above the disk surface with no pole tip protrusion. (b) Summary plot showing the lube mogul height as a function of Z-Tetraol 2200 film thickness (blue circles are taken from [6]).

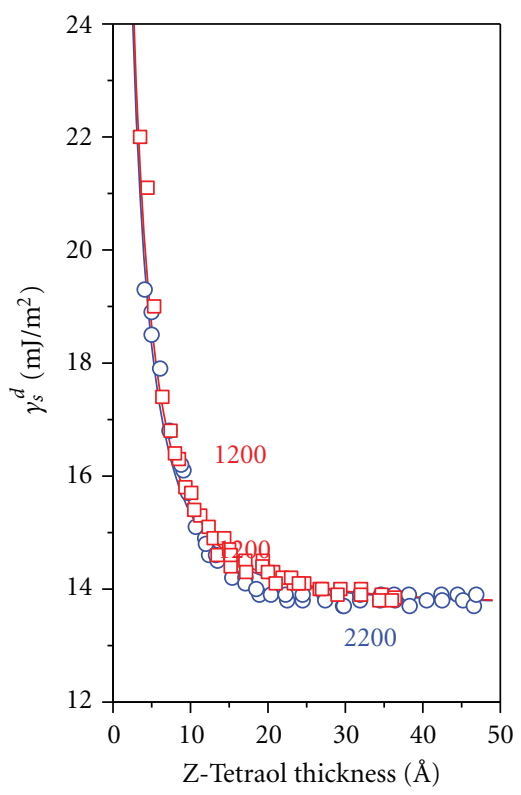

(a)

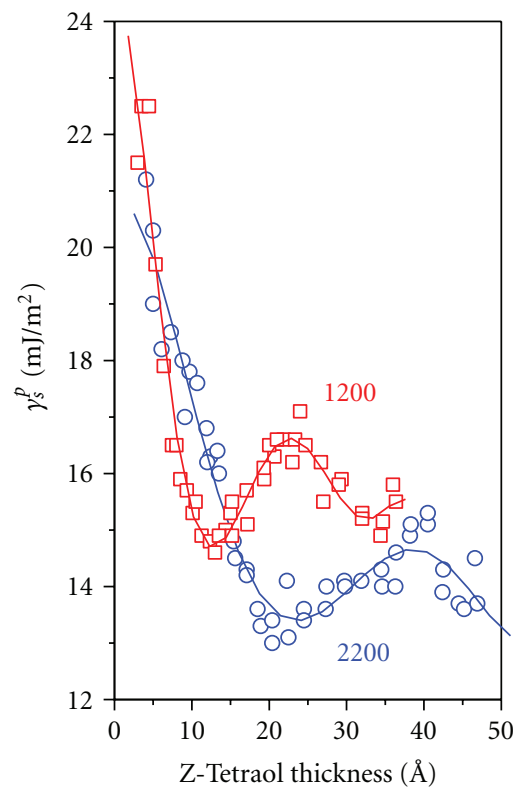

(b)

Figure 4: (a) Dispersive $\left(\gamma_{s}^{d}\right)$ and (right) polar $\left(\gamma_{s}^{p}\right)$ surface energy as a function of Z-Tetraol film thickness and MW. 


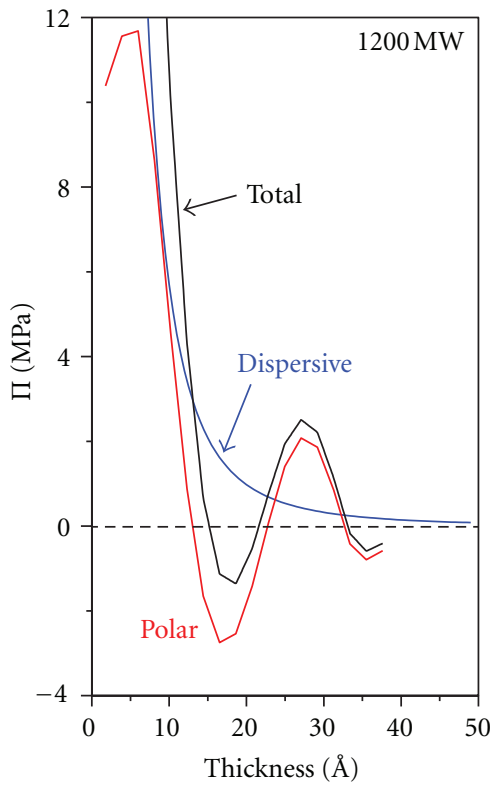

(a)

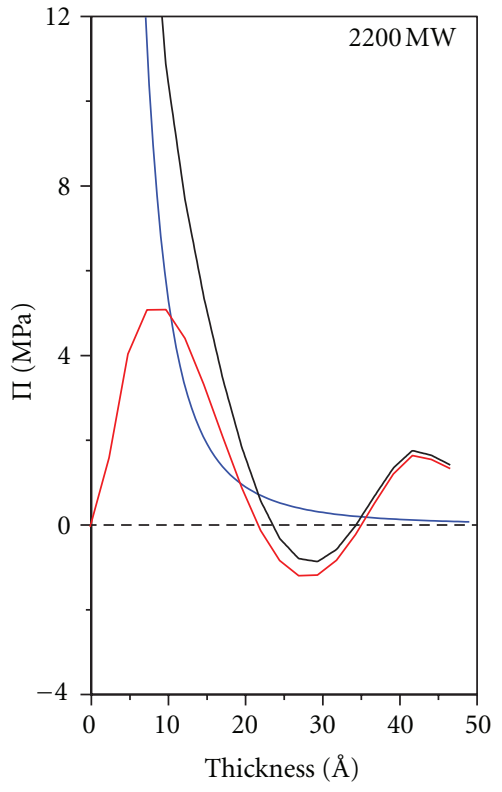

(b)

Figure 5: Surface energy-derived disjoining pressure (П) as a function of Z-Tetraol film thickness.

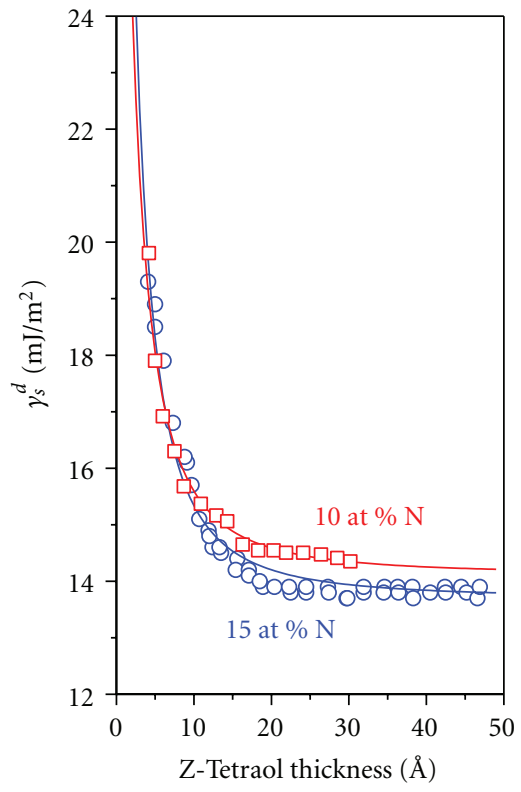

(a)

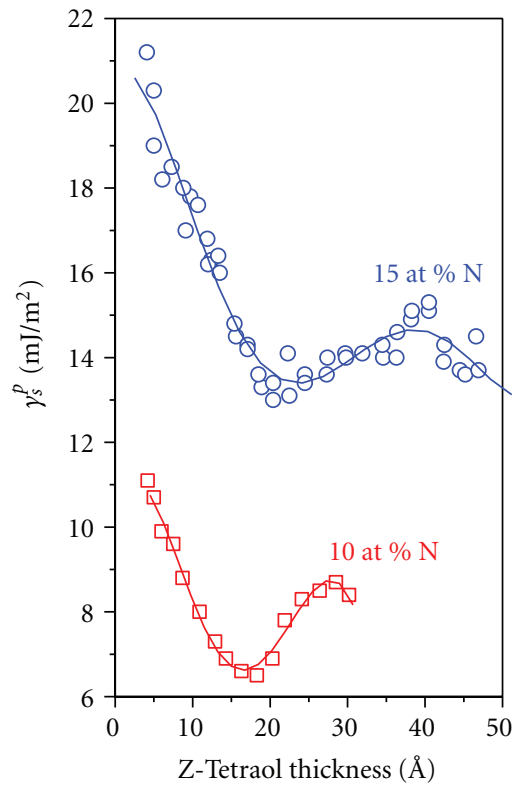

(b)

FIgURE 6: (a) Dispersive $\left(\gamma_{s}^{d}\right)$ and (b) polar $\left(\gamma_{s}^{p}\right)$ surface energy of Z-Tetraol 2200 as a function of film thickness and carbon film composition.

Therefore the lower MW film is expected to be more resistant to lubricant disturbance and moguls formation for thinner films ( $\leq \sim 10 \AA)$. However, since the monolayer thickness of lower MW Z-Tetraol is also thinner, the ability to resist film disturbance will decrease more rapidly than higher MW Z-Tetraol as the disjoining pressure converges towards zero. This would occur at approximately 14 and $22 \AA$ for Z-Tetraol 1200 and 2200, respectively, (Figure 5). Thus the film thickness employed at the MW of choice is a significant determinant. The monolayer thicknesses for Z-Tetraol 1200 and 2200 , very useful quantities indeed, are summarized in Table 2.

A relevant observation regarding the quantification of the Z-Tetraol monolayer thickness by surface energy measurements is herewith disclosed. In particular, it has a pronounced dependence on the composition of the underlying carbon film. Figure 6 shows that the Z-Tetraol monolayer film thickness increases with increasing atomic $\% \mathrm{~N}$, as 


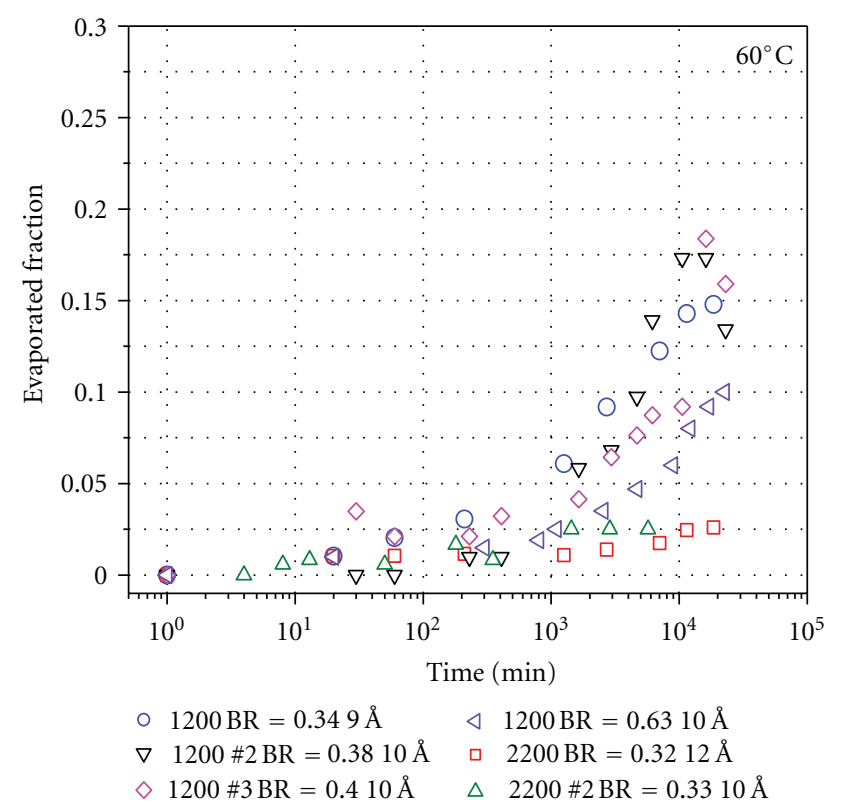

FIgURE 7: Evaporative loss of 9-12 $\AA$ Z-Tetraol films on $\mathrm{CN}_{x}$ at $60^{\circ} \mathrm{C}$. The experiments were repeated more than several times as a check of consistency. The inset legend specifies the MW (1200 or 2200), the bonded ratio (BR) when the experiment was begun, and the initial film thickness (9-12 $\AA$ ). The bonded ratio was varied by disk aging under ambient conditions; that is, no annealing or UV treatment was applied to the disks.

TABLE 2: Monolayer thicknesses for Z-Tetraols on $\mathrm{CN}_{x}(\AA)$.

\begin{tabular}{lcc}
\hline Z-Tetraol & $\begin{array}{c}\gamma_{s}^{p} \\
\text { (Figure 4) }\end{array}$ & $\begin{array}{c}\Pi=0 \mathrm{MPa} \\
\text { (Figure 5) }\end{array}$ \\
\hline 1200 & 13 & 14 \\
2200 & 20 & 23 \\
\hline
\end{tabular}

previously observed for Zdol [11]. We therefore note that the film thickness of choice in HDDs can be influenced by factors other than the PFPE MW itself, and some compensation by the underlying film appears to be possible.

Another limitation of utilizing lower MW lubricants is the increased evaporative loss expected under HDD conditions. Figure 7 shows the film thickness loss as a function of time and of Z-Tetraol MW near the HDD temperature of $60^{\circ} \mathrm{C}$. With decreasing MW, significant film thickness loss can occur leading to degraded performance in the HDD due to the lack of lubricant film coverage. Postlubrication treatment such as annealing or UV curing may be employed to improve the bonded fraction and hence reduce evaporative loss of lower MW components in the lubricant film. Figure 7 shows decreased evaporation with increased initial bonded ratio (BR).

\section{Conclusion}

Lower MW Z-Tetraol films at the same total film thickness provide more mechanical clearance than higher MW ZTetraol due to its smaller $z$-profile. The increased resistance to lubricant disturbance on the disk surface (e.g., lube moguls) is attributed to its increased molecular polarity; however, this behavior is strongly film thickness dependent and approaching the monolayer will quickly dissipate this advantage. The monolayer film thickness for the same ZTetraol MW may be manipulated by either increasing or decreasing the nitrogen content of the underlying carbon film.

\section{Acknowledgments}

The authors thank J. Burns and D. Pocker of this laboratory for the NMR and ESCA data, respectively.

\section{References}

[1] A. Khurshudov and R. J. Waltman, "The contribution of thin PFPE lubricants to slider-disk spacing," Tribology Letters, vol. 11, no. 3-4, pp. 143-149, 2001.

[2] R. J. Waltman and A. G. Khurshudov, "The contribution of thin PFPE lubricants to slider-disk spacing. 2. Effect of film thickness and lubricant end groups," Tribology Letters, vol. 13, no. 3, pp. 197-202, 2002.

[3] R. J. Waltman, V. Raman, and J. Burns, "The contribution of thin PFPE lubricants to slider-disk spacing. 3. Effect of main chain flexibility," Tribology Letters, vol. 17, no. 2, pp. 239-244, 2004.

[4] M. F. Toney, C. M. Mate, and D. J. Pocker, "Calibrating ESCA and ellipsometry measurements of perfluoropolyether lubricant thickness," IEEE Transactions on Magnetics, vol. 34, no. 4, pp. 1774-1776, 1998.

[5] Q. Dai, C. Saint-Olive, R. Pit, and B. Marchon, "Genesis and evolution of lubricant moguls," IEEE Transactions on Magnetics, vol. 38, no. 5 I, pp. 2111-2113, 2002. 
[6] R. J. Waltman, H. Deng, G. J. Wang, H. Zhu, and G. W. Tyndall, "The effect of PFPE film thickness and molecular polarity on the pick-up of disk lubricant by a low-flying slider," Tribology Letters, vol. 39, no. 2, pp. 211-219, 2010.

[7] J. N. Israelachvili, Intermolecular and Surface Forces with Applications to Colloidal and Biological Systems, Academic Press, London, UK, 1985.

[8] G. W. Tyndall, T. E. Karis, and M. S. Jhon, "Spreading profiles of molecularly thin perfluoropolyether films," Tribology Transactions, vol. 42, no. 3, pp. 463-470, 1999.

[9] A. Y. Suh and A. A. Polycarpou, "Adhesive contact modeling for sub-5-nm ultralow flying magnetic storage head-disk interfaces including roughness effects," Journal of Applied Physics, vol. 97, no. 10, Article ID 104328, 11 pages, 2005.

[10] C. M. Mate, "Taking a fresh look at disjoining pressure of lubricants at slider-disk interfaces," IEEE Transactions on Magnetics, vol. 47, no. 1, pp. 124-130, 2011.

[11] R. J. Waltman and G. W. Tyndall, "Lubricant and overcoat systems for rigid magnetic recording media," Journal of the Magnetics Society of Japan, vol. 26, no. 3, pp. 97-108, 2002. 

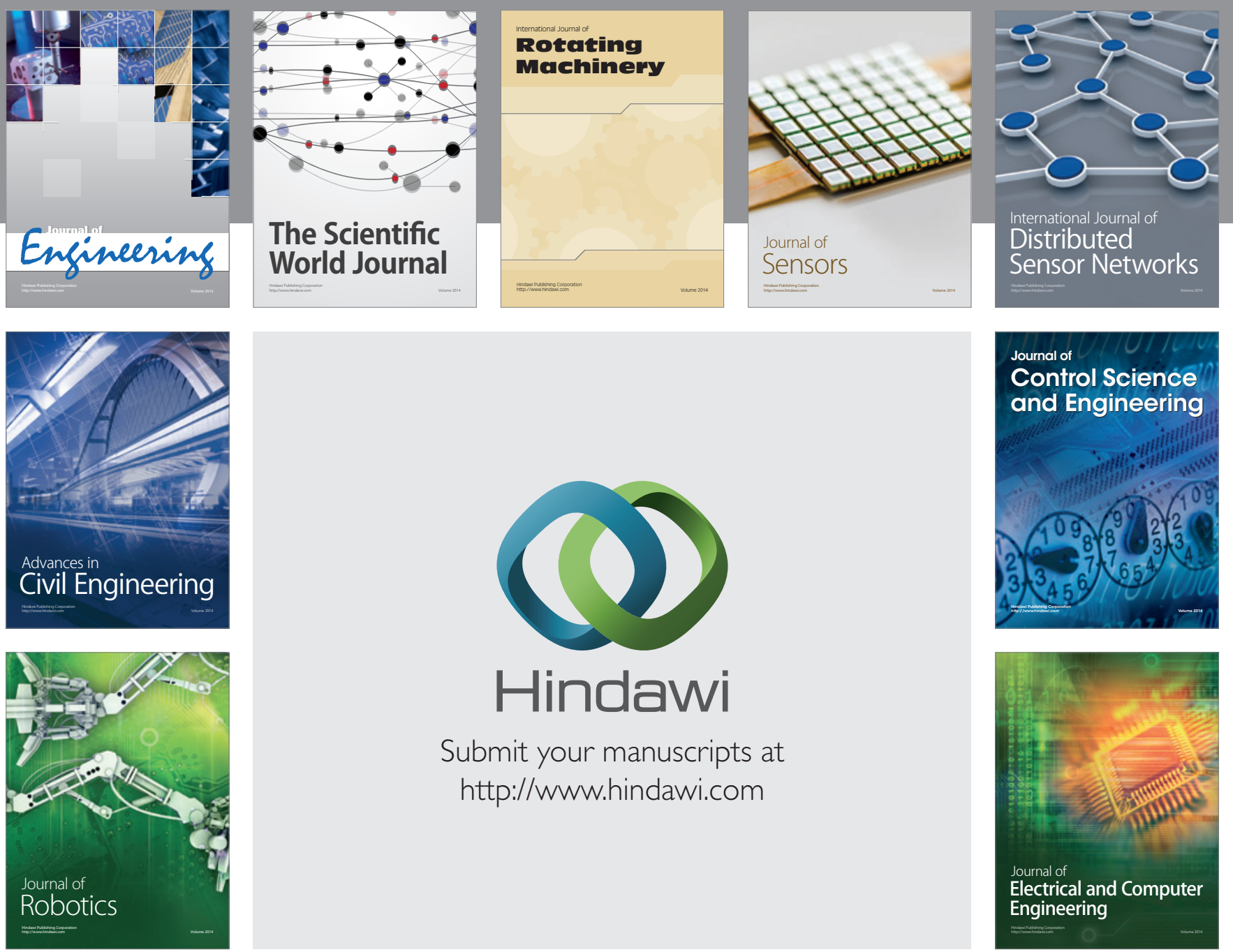

Submit your manuscripts at

http://www.hindawi.com
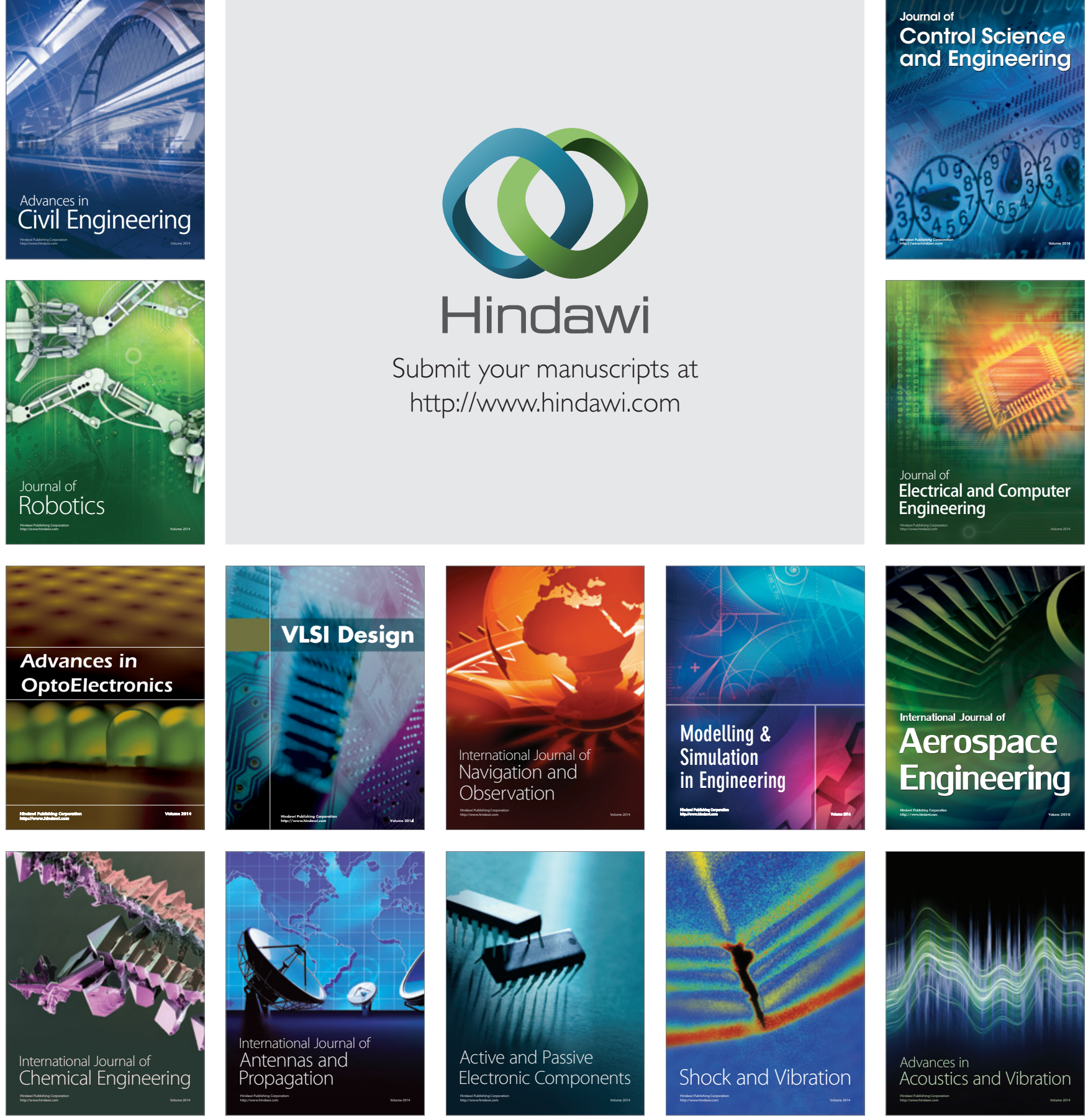